\title{
On Soft Generalized Closed Sets in a Soft Topological Space with a Soft Weak Structure
}

Hanan S. Al-Saadi ${ }^{1}$ and Won Keun Min $^{2}$

${ }^{1}$ Department of Mathematics, Faculty of Applied Sciences, Umm Al-Qura University, Makkah, Saudi Arabia

${ }^{2}$ Department of Mathematics, Kangwon National University, Chuncheon, Korea

\begin{abstract}
In this work, we introduce the notion of generalized $\omega$-closed set in a soft topological space with a soft weak structure. And some basic properties of this new class are investigated by using the concept of weak structure. Moreover, we study soft $\omega$ - $T_{\frac{1}{2}}$-spaces defined by soft $g \omega$-closed sets and study some properties of it by using $g \omega$-open sets.
\end{abstract}

Keywords: Soft set, Soft topology, Soft weak structure, Soft generalized closed set

\section{Introduction}

As a solution to many problems, scientists have resorted to use approximate solution. In 1999, Molodtsove [1] initiated the concept of soft set theory as a new mathematical tool which is free from the problems mentioned above. Later on Maji et al [2] proposed several operations on soft sets and some basic properties and then Pei and Miao [3] investigated the relationships between soft sets and information systems.

In 2011, Shabir and Naz [4] introduced the notion of soft topological spaces and Min [5] corrected some their results. Zorlutuna et al. [6] continued to study the properties of soft topological spaces by defining the concepts of interior, soft neighborhoods in soft topological spaces. Varo and Aygun [7] presented soft Hausdorff spaces and introduced some new concepts such as convergence of sequences. Levine [8] introduced generalized closed sets in topological spaces.

In 2013, Cagman et al. [9] defined soft topological spaces by modifying the soft set. Also,

Received: Dec. 12, 2017

Revised : Dec. 20, 2017

Accepted: Dec. 27, 2017

Correspondence to: Won Keun Min (wkmin@kangwon.ac.kr)

(CThe Korean Institute of Intelligent Systems

(c) This is an Open Access article distributed under the terms of the Creative Commons Attribution Non-Commercial License (http://creativecommons.org/licenses/ by-nc/3.0// which permits unrestricted noncommercial use, distribution, and reproduction in any medium, provided the original work is properly cited. Roy and Samanta [10] strengthen the definition of the soft topological spaces presented in [9] and they used the base and the subbase to characterize its properties.

In 1997, Csaszar [4] has studied generalized topological notions in collections which are closed under unions. Many other authors [12,-15] investigated the properties of the generalized topology. Recently, Császár [16] introduced a new notion called weak structures. Let $X$ be a non-empty set and $P(X)$ be its power set. A structure $\omega$ on $X$ is called a weak structure (briefly, $W S$ ) on $X$ if and only if $\phi \in \omega[16]$.

In 2012, Al-Omari and Noiri [17] introduced and studied a kind of sets called generalized $\omega$ closed (briefly, $g \omega$-closed) sets in topological space. In this paper, we introduce the notions of soft generalized $\omega$-closed (briefly, soft $g \omega$-closed) sets and soft $g \omega$-open sets in soft topological spaces. Also, we will investigate some new soft separation axioms. In particular, we study soft $\omega-T_{\frac{1}{2}}$-spaces. 
Let $X$ be a non-empty set, $E$ a set of parameters, $P(X)$ denote the power set of $X$ and $A$ be a non-empty subset of $E$.

Definition 1.1 ( [1]]). For $A \subseteq E$, a pair $(F, A)$ is called a soft set over $X$, where $F$ is a mapping given by $F: A \rightarrow P(X)$. For $e \in A, F(e)$ may be considered as the set of $e$-approximate elements of the soft set $(F, A)$.

Definition $1.2([5])$. A soft set $(F, A)$ over $X$ is said to be:

1. A null soft set denoted by $\tilde{\emptyset}$ if $F(e)=\emptyset$ for all $e \in A$.

2. An absolute soft set denoted by $\tilde{X}$ if $F(e)=X$ for all $e \in A$.

Definition 1.3 ( [2, 5, 18]). For any two soft sets $(F, A)$ and $(G, B)$ defined over a common universe $X$, we have:

1. $(F, A) \tilde{\complement}(G, B)$ iff $A \subseteq B$ and $F(e) \subseteq G(e)$ for all $e \in A$.

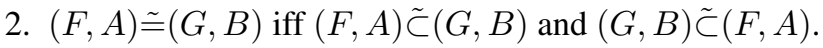

3. $(F, A) \tilde{\cup}(G, B) \tilde{=}(H, C)$ where $C=A \cup B$ and

$$
H(e)= \begin{cases}F(e), & \text { if } e \in A-B, \\ G(e), & \text { if } e \in B-A, \\ F(e) \cup G(e), & \text { if } e \in A \cap B .\end{cases}
$$

for all $e \in C$.

4. $(F, A) \tilde{\cap}(G, B) \tilde{=}(K, D)$ where $D=A \cap B$ and $K(e)=$ $F(e) \cap G(e)$ for all $e \in C$.

5. $x \in(F, A)$ where $x \in X$ iff $x \in F(e)$ for all $e \in A$ and $x \notin(F, A)$ whenever $x \notin F(e)$ for some $e \in A$.

(6) $(F, E) \tilde{-}(G, E) \simeq(M, E)$ where $M(e)=F(e)-G(e)$ for all $e \in E$.

Definition 1.4 ( [19]). For a soft set $(F, A)$ over $X$, the relative complement of $(F, A)$ (denoted by $\left.(F, A)^{\prime}\right)$ is defined by $(F, A)^{\prime} \tilde{=}\left(F^{\prime}, A\right)$, where $F^{\prime}: A \rightarrow P(X)$ is given by $F^{\prime}(e)=X-F(e)$ for all $e \in A$.

Definition 1.5 ( [4]). Let $\tau$ be the collection of soft sets over $X$, then $\tau$ is called a soft topology on $X$ if $\tau$ satisfies the following axioms:

1. $\tilde{\emptyset}, \tilde{X}$ belong to $\tau$.

2. The union of any number of soft sets in $\tau$ belong to $\tau$.
3. The intersection of any two soft sets in $\tau$ belong to $\tau$.

The triple $(X, \tau, E)$ is called a soft topological space over $X$. The member of $\tau$ are said to be soft open set in $X$. A soft set $(F, E)$ over $X$ is said to be soft closed in $X$ if its relative complement $(F, E)^{\prime}$ belong to $\tau$.

Lemma 1.6 ([14] ). Let $(F, E)$ be a soft set over $X$ and $x \in X$, then

1. $x \in(F, E)$ iff $(x, E) \tilde{C}(F, E)$

2. if $(x, E) \cap(F, E)=\emptyset$, then $x \notin(F, E)$.

Csaszar defined $i_{\omega}(A)$ as the union of all $\omega$-open subsets of $A$ and $c_{\omega}(A)$ as the intersection of all $\omega$-closed sets containing $A$.

Let $X$ be a non-empty set and $E$ be a set of parameters. A collection $\omega$ of soft sets defined over $X$ with respect to $E$ is called a soft weak structure [20] iff $\tilde{\emptyset} \in \omega$. A soft set $(F, A)$ is called $\omega$-open soft set iff $(F, A) \in \omega$ and called $\omega$-closed soft set iff $(F, A)^{\prime} \in \omega$.

Definition 1.7 ( [19]). In a soft topological spaces $(X, \tau, E)$, a soft set $(F, E)$ over $X$ is called a soft generalized closed set (briefly, soft g-closed) if $C l(F, E) \subseteq(G, E)$ whenever $(F, E) \subseteq(G, E)$ and $(G, E)$ is soft open in $(X, \tau, E)$.

Lemma 1.8 ([20]). Let $\omega$ be a soft weak structure defined over $X$ with respect to $E$ and $(F, E),(G, E)$ are two soft sets over $X$, then:

1. $i_{\omega}(F, E) \tilde{\complement}(F, E)$

2. If $(F, E) \tilde{C}(G, E)$, then $i_{\omega}(F, E) \tilde{\subset} i_{\omega}(G, E)$ and $c_{\omega}(F, E)$ $\tilde{\subset} c_{\omega}(G, E)$

3. $i_{\omega}\left(i_{\omega}(F, E)\right) \tilde{=} i_{\omega}(F, E)$ and $c_{\omega}\left(c_{\omega}(F, E)\right) \tilde{=} c_{\omega}(F, E)$.

4. $i_{\omega}(F, E)^{\prime}=\left(c_{\omega}(F, E)\right)^{\prime}$ and $c_{\omega}(F, E)^{\prime}=\left(i_{\omega}(F, E)\right)^{\prime}$.

Lemma 1.9 ( [20]). Let $\omega$ be a soft weak structure defined over $X$ with respect to the parameters set $E$ and $(F, E)$ be a soft set, then:

1. $x \in i_{\omega}(F, E)$ if there exists an $\omega$-open soft set $(\mathrm{G}, \mathrm{E})$ such that $x \in(G, E) \tilde{\subset}(F, E)$.

2. $x \in c_{\omega}(F, E)$ if and only if $(G, E) \tilde{\cap}(F, E) \neq \tilde{\emptyset}$ for all $(G, E) \in \omega$ such that $x \in(G, E)$.

3. If $(F, E) \in \omega$, then $(F, E)=i_{\omega}(F, E)$ and if $(F, E)$ is $\omega$-closed soft set, then $(F, E)=c_{\omega}(F, E)$ 
Lemma 1.10 ( [4]). Let $(X, \tau, E)$ be a soft topological space over $X$. If $(x, E)$ is a soft closed set in $\tau$ for each $x \in X$, then $(X, \tau, E)$ is a soft $T_{1}$-space.

Definition 1.11 ( [20]). A soft topological space $(X, \tau, E)$ is called:

1. Soft $\omega-T_{0}$ if for each $x, y \in X$ such that $x \neq y$, there exists a soft $\omega$-open set $(F, E)$ such that $x \in(F, E)$ and $y \notin(F, E)$ or $x \notin(F, E)$ and $y \in(F, E)$.

2. Soft $\omega-T_{1}$ if for each $x, y \in X$ such that $x \neq y$, there exists soft $\omega$-open sets $(F, E)$ and $(G, E)$ such that $x \in$ $(F, E), y \notin(F, E), x \notin(G, E)$ and $y \in(G, E)$.

\section{Soft $g \omega$-Closed Set}

Definition 2.1. Let $X$ be a non-empty set and $E$ be a set of parameters. Let a collection $\omega$ be a soft weak structure on a soft topological space $(X, \tau, E)$. Then a soft set $(F, E)$ over $X$ is called a soft generalized $\omega$-closed set (briefly, soft $g \omega$-closed set) if $c_{\omega}(F, E) \tilde{\complement}(G, E)$ whenever $(F, E) \tilde{\complement}(G, E)$ and $(G, E)$ is soft open in $(X, \tau, E)$. The complement of a soft $g \omega$-closed set is called a soft generalized $\omega$-open (briefly, soft $g \omega$-open) set.

Example 2.2. Let $X=\left\{h_{1}, h_{2}, h_{3}\right\}, E=\left\{e_{1}, e_{2}\right\}$ and $\tau=$ $\left\{\tilde{\emptyset}, \tilde{X},\left(F_{1}, E\right),\left(F_{2}, E\right),\left(F_{3}, E\right),\left(F_{4}, E\right)\right\}$ where

$$
\begin{array}{cccc}
F_{1}\left(e_{1}\right)=\left\{h_{2}\right\}, & F_{1}\left(e_{2}\right)= & \left\{h_{1}\right\} ; \\
F_{2}\left(e_{1}\right)= & \left\{h_{2}, h_{3}\right\}, & F_{2}\left(e_{2}\right)= & \left\{h_{1}, h_{2}\right\} ; \\
F_{3}\left(e_{1}\right)= & \left\{h_{1}, h_{2}\right\}, & F_{3}\left(e_{2}\right)= & \left\{h_{1}, h_{3}\right\} ; \\
F_{4}\left(e_{1}\right)= & \left\{h_{1}\right\}, & F_{4}\left(e_{2}\right)= & \left\{h_{3}\right\} .
\end{array}
$$

Let $\omega=\left\{\tilde{\emptyset},\left(F_{1}, E\right),\left(F_{3}, E\right)\right\}$ be a soft weak structure over $X$ with respect to $E$. Then $(G, E)$ is a soft $g \omega$-closed defined by $G\left(e_{1}\right)=\tilde{\emptyset} ; G\left(e_{2}\right)=\left\{h_{2}\right\}$, but $\left(F_{1}, E\right)$ is not soft $g \omega$-closed.

Remark 2.3. Let $\omega$ be a soft weak structure on a soft topological space $(X, \tau, E)$. Then every soft $g \omega$-closed set is soft $g$-closed. The following example shows that the converse need not be true in general.

Example 2.4. In Example 2.2. $\left(F_{2}, E\right)$ is soft $g$-closed in $(X$, $\tau, E)$ but not soft $g \omega$-closed over $X$.

Remark 2.5. For a soft weak structure $\omega$ on a soft topological space over $X$, every $\omega$-closed set is a soft $g \omega$-closed set. In fact, if $(F, E)$ is a $\omega$-closed set $(F, E) \tilde{C}(G, E)$ and $(G, E)$ is soft open, then $(F, E)=c_{\omega}(F, E) \tilde{\subset}(G, E)$, so that $(F, E)$ is soft $g \omega$-closed. The following example shows that the converse need not be true in general.

Example 2.6. In Example 2.2, $(G, E)$ is soft $g \omega$-closed but not $\omega$-closed.

Theorem 2.7. Let $\omega$ be a soft weak structure on a soft topological space $(X, \tau, E)$. If $(F, E)$ is soft $g \omega$-closed in $X$ and $(F, E) \tilde{\subset}(H, E) \tilde{\subset} c_{\omega}(F, E)$, then $(H, E)$ is soft $g \omega$-closed

Proof. Suppose that $(F, E)$ is soft $g \omega$-closed over $X$ and $(F, E)$ $\tilde{\subset}(H, E) \tilde{\subset} c_{\omega}(F, E)$. Let $(H, E) \tilde{C}(G, E)$ and $(G, E)$ is soft open in $X$. Since $(F, E) \tilde{\subset}(H, E)$ and $(H, E) \tilde{\subset}(G, E)$, we have $(F, E) \tilde{C}(G, E)$. Since $(F, E)$ is soft $g \omega$-closed, then $c_{\omega}(A, E) \tilde{\subset}(G, E)$. Since $(H, E) \tilde{\subset} c_{\omega}(F, E)$, we have $c_{\omega}(H, E)$ $\tilde{\subset} c_{\omega}(F, E) \tilde{\subset}(G, E)$. Therefore $(H, E)$ is soft $g \omega$-closed.

The next example shows that the intersection of two soft $g \omega$-closed sets is not in general soft $g \omega$-closed.

Example 2.8. Let $X=\left\{h_{1}, h_{2}, h_{3}\right\}, E=\left\{e_{1}, e_{2}\right\}$ and $\tau=$ $\left\{\tilde{\emptyset}, \tilde{X},\left(F_{1}, E\right),\left(F_{2}, E\right),\left(F_{3}, E\right),\left(F_{4}, E\right)\right\}$ where

$$
\begin{array}{lllc}
F_{1}\left(e_{1}\right)= & \left\{h_{1}\right\}, & F_{1}\left(e_{2}\right)= & \left\{h_{2}\right\} ; \\
F_{2}\left(e_{1}\right)=\left\{h_{3}\right\}, & F_{2}\left(e_{2}\right)= & \left\{h_{1}, h_{3}\right\} ; \\
F_{3}\left(e_{1}\right)= & \left\{h_{1}, h_{2}\right\}, & F_{3}\left(e_{2}\right)= & \left\{h_{2}, h_{3}\right\} ; \\
F_{4}\left(e_{1}\right)= & \left\{h_{1}, h_{3}\right\}, & F_{4}\left(e_{2}\right)= & \emptyset .
\end{array}
$$

Let $\omega=\left\{\tilde{\emptyset},\left(F_{2}, E\right)\right\}$ be a soft weak structure over $X$ with respect to $E$. Then $(H, E)$ be a soft $g \omega$-closed defined by $H\left(e_{1}\right)=H\left(e_{2}\right)=\left\{h_{2}\right\}$. Thus it can be easily checked that $\left(F_{1}, E\right) \tilde{\cap}(H, E)$ is not a soft $g \omega$-closed set.

Theorem 2.9. Let $\omega$ be a soft weak structure on a soft topological space $(X, \tau, E)$. If $(F, E)$ is a soft $g \omega$-closed set, then $c_{\omega}(F, E)-(F, E)$ does not contain any non-empty soft closed set.

Proof. Let $(H, E)$ be a soft closed subset of $X$ such that $(H, E)$ $\tilde{\complement} c_{\omega}(F, E)-(F, E)$, where $(F, E)$ is soft $g \omega$-closed. Since $(H, E)^{\prime}$ is soft open, $(F, E) \tilde{C}(H, E)^{\prime}$ and $(F, E)$ is soft $g \omega$ closed, $c_{\omega}(F, E) \tilde{\complement}(H, E)^{\prime}$ and thus $(H, E) \tilde{\complement}\left[c_{\omega}(F, E)\right]^{\prime}$. Thus $(H, E) \tilde{\complement}\left[c_{\omega}(F, E)\right]^{\prime} \tilde{\cap} c_{\omega}(F, E)=\tilde{\emptyset}$ and hence $(H, E)=\tilde{\emptyset}$.

If $c_{\omega}(F, E)-(F, E)$ does not contain any non-empty soft closed subset of $X$, then $(F, E)$ need not be soft $g \omega$-closed in general.

Example 2.10. In Example 2.8, let $(G, E)$ be a soft set defined by $G\left(e_{1}\right)=\tilde{\emptyset}, G\left(e_{2}\right)=\left\{h_{2}\right\}$. Then $c_{\omega}(G, E)-(G, E)=$ 
$(K, E)$ does not contain any non empty soft closed set such that $K\left(e_{1}\right)=\left\{h_{1}, h_{2}\right\}, K\left(e_{2}\right)=\emptyset$, but $(G, E)$ is not a soft $g \omega$-closed set.

Corollary 2.11. Let $\omega$ be a soft weak structure on a soft topological space $(X, \tau, E)$ and $(F, E)$ be a soft $g \omega$-closed set. Then $c_{\omega}(F, E)=(F, E)$ if and only if $c_{\omega}(F, E)-(F, E)$ is soft closed.

Proof. Let $(F, E)$ be a soft $g \omega$-closed set. If $c_{\omega}(F, E)=$ $(F, E)$, then $c_{\omega}(F, E)-(F, E)=\emptyset$, and $c_{\omega}(F, E)-(F, E)$ is a soft closed set.

Conversely, let $c_{\omega}(F, E)-(F, E)$ be a soft closed set. Since $(F, E)$ is soft $g \omega$-closed, then by Theorem 2.9. $c_{\omega}(F, E)-$ $(F, E)$ does not contain any non-empty soft closed set. Since $c_{\omega}(F, E)-(F, E)$ is a soft closed subset of itself, $c_{\omega}(F, E)-$ $(F, E)=\emptyset$ and hence $c_{\omega}(F, E)=(F, E)$.

Theorem 2.12. Let $\omega$ be a soft weak structure on a soft topological space $(X, \tau, E)$. Then $(H, E)$ is soft $g \omega$-open if and only if $(F, E) \tilde{\subset} i_{\omega}(H, E)$ whenever $(F, E) \tilde{\subset}(H, E)$ and $(F, E)$ is soft closed.

Proof. Let $(H, E)$ be a soft $g \omega$-open set and $(F, E) \tilde{\subset}(H, E)$, where $(F, E)$ is soft closed. Then $(H, E)^{\prime}$ is a soft $g \omega$-closed set contained in a soft open set $(F, E)^{\prime}$. Hence $c_{\omega}(H, E)^{\prime}$ $\tilde{\subset}(F, E)^{\prime}$, i.e. $\left(i_{\omega}(H, E)\right)^{\prime} \tilde{\subset}(F, E)^{\prime}$. So $(F, E) \tilde{\subset} i_{\omega}(H, E)$.

Conversely, Suppose that $(F, E) \tilde{\subset} i_{\omega}(H, E)$ for any soft closed set $(F, E)$ whenever $(F, E) \tilde{\subset}(H, E)$. Let $(H, E)^{\prime} \tilde{\subset}(G, E)$, where $(G, E)$ is a soft open set. Then $(G, E)^{\prime} \tilde{\subset}(H, E)$ and $(G, E)^{\prime}$ is soft closed. By assumption, $(G, E)^{\prime} \tilde{\subset} i_{\omega}(H, E)$ and hence $c_{\omega}(H, E)=\left(i_{\omega}(H, E)\right)^{\prime} \tilde{\subset}(G, E)$. Therefore, $(H, E)^{\prime}$ is soft $g \omega$-closed and hence $(H, E)$ is soft $g \omega$-open.

Theorem 2.13. Let $\omega$ be a soft weak structure on a soft topological space $(X, \tau, E)$. Then the following are equivalent:

1. For every soft open $(G, E)$ of $X, c_{\omega}(G, E) \tilde{\subset}(G, E)$.

2. Every soft subset of $X$ is soft $g \omega$-closed.

Proof. (1) $\Longrightarrow(2)$ Let $(F, E)$ be any soft subset of $X$ where $(F, E) \tilde{\subset}(G, E)$ and $(G, E)$ is any soft open set. Then by (1), $c_{\omega}(G, E) \tilde{\subset}(G, E)$ and hence $c_{\omega}(F, E) \tilde{\subset} c_{\omega}(G, E) \tilde{\subset}(G, E)$.

Thus $(F, E)$ is soft $g \omega$-closed.

$(2) \Longrightarrow(1)$ Let $(G, E)$ be any soft open set. Then by $(2),(G, E)$ is soft $g \omega$-closed and hence $c_{\omega}(G, E) \tilde{\subset}(G, E)$.

Theorem 2.14. Let $\omega$ be a soft weak structure on a soft topological space $(X, \tau, E)$. If a soft set $(H, E)$ is soft $g \omega$-open and if for a soft open set $(G, E), i_{\omega}(H, E) \tilde{\cup}(H, E)^{\prime} \tilde{\subset}(G, E)$, then $(G, E)^{\prime}=\tilde{\emptyset}$.

Proof. Let $(G, E)$ be a soft open set and $i_{\omega}(H, E) \tilde{\cup}(H, E)^{\prime} \tilde{\subset}$ $(G, E)$ for a soft $g \omega$-open set $(H, E)$. Then $(G, E)^{\prime} \tilde{\subset}\left(i_{\omega}(H, E)\right)^{\prime}$ $\tilde{\cap}(H, E)$. This implies $(G, E)^{\prime} \tilde{\subset} c_{\omega}(H, E)-(H, E)$. Since $(H, E)^{\prime}$ is soft $g \omega$-closed, by Theorem 2.9. $(G, E)^{\prime}=\tilde{\emptyset}$.

Theorem 2.15. Let $\omega$ be a soft weak structure on a soft topological space $(X, \tau, E)$. If a soft set $(H, E)$ is soft $g \omega$-open and $i_{\omega}(H, E) \tilde{\subset}(K, E) \tilde{\subset}(H, E)$, then $(K, E)$ is soft $g \omega$-open.

Proof. We have $(H, E)^{\prime} \tilde{\subset}(K, E)^{\prime} \tilde{\subset} c_{\omega}(H, E)^{\prime}$. Since $(H, E)$ is soft $g \omega$-closed, from Theorem 2.7, it follows that $(K, E)^{\prime}$ is soft $g \omega$-closed, and hence $(K, E)$ is soft $g \omega$-open.

\section{Separation Axioms}

In this section, we introduce the new separation axiom, namely soft $\omega-T_{\frac{1}{2}}$-space in soft topological space with a soft weak structure $\omega$.

Definition 3.1. A soft topological space $(X, \tau, E)$ with a soft weak structure $\omega$ is called a soft $\omega-T_{\frac{1}{2}}$-space if for every soft $g \omega$-closed set $(F, E)$ of $X, c_{\omega}(F, E)=(F, E)$

Theorem 3.2. Let $\omega$ be a soft weak structure on $X$. A soft topological space $(X, \tau, E)$ is soft $\omega-T_{1}$ if $(x, E)$ is soft $\omega$ closed set for all $x \in X$.

Proof. Let $x, y \in X$ such that $x \neq y$. Then $(x, E)^{\prime}$ and $(y, E)^{\prime}$ are soft $\omega$-open sets such $y \in(x, E)^{\prime}, x \notin(x, E)^{\prime}$ and $y \notin$ $(y, E)^{\prime}, x \in(y, E)^{\prime}$. Hence $X$ is soft $\omega-T_{1}$.

Corollary 3.3. If the union of soft $\omega$-open sets is soft $\omega$-open set, then the converse of Theorem 3.2, is true.

Theorem 3.4. Let $\omega$ be a soft weak structure on a soft topological space $(X, \tau, E)$ with respect to $E$. Then the following statements are equivalent:

1. $X$ is a soft $\omega-T_{\frac{1}{2}}$-space.

2. Every singleton is either soft closed or $(x, E)=i_{\omega}(x, E)$

Proof. $(1) \Longrightarrow(2)$. Suppose $(x, E)$ is not a soft closed subset for some $x \in X$. Then $(x, E)^{\prime}$ is not soft open, so if there is, then $X$ is the only soft open set containing $(x, E)^{\prime}$. Therefore $(x, E)^{\prime}$ is soft $g \omega$-closed. Since $X$ is soft $\omega-T_{\frac{1}{2}}-$ space, $c_{\omega}(x, E)^{\prime}=\left(i_{\omega}(x, E)\right)^{\prime}=(x, E)^{\prime}$ and thus $(x, E)=$ $i_{\omega}(x, E)$. 
(2) $\Longrightarrow(1)$ Let $(F, E)$ be a soft $g \omega$-closed subset of $X$ and $x \in c_{\omega}(F, E)$. We show that $x \in(F, E)$. If $(x, E)$ is soft closed and $x \notin(F, E)$, then $x \in\left(c_{\omega}(F, E)-(F, E)\right)$. Then $(x, E) \tilde{\subset}(F, E)^{\prime}$ and hence $(F, E) \tilde{\subset}(x, E)^{\prime}$. Since $(F, E)$ is a soft $g \omega$-closed set and $(x, E)^{\prime}$ is a soft open, $c_{\omega}(F, E) \tilde{\subset}(x, E)^{\prime}$ and hence $(x, E) \tilde{\subset}\left(c_{\omega}(F, E)\right)^{\prime}$. Therefore, $(x, E) \tilde{\subset} c_{\omega}(F, E)$ $\tilde{\cap}\left(c_{\omega}(F, E)\right)^{\prime}=\tilde{\emptyset}$. This is a contradiction. Therefore, $x \in$ $(F, E)$. If $(x, E)=i_{\omega}(x, E)$, since $x \in c_{\omega}(F, E)$, then for every soft $\omega$-open set $(G, E)$ such that $x \in(G, E)$, we have $(G, E) \tilde{\cap}(F, E) \neq \tilde{\emptyset}$. By assumption $(x, E)=i_{\omega}(x, E)$, we have $(x, E)$ be an $\omega$-open soft set and $(x, E) \tilde{\cap}(F, E) \neq \tilde{\emptyset}$. Hence $x \in(F, E)$. Therefore, in both cases we have $x \in$ $(F, E)$. Therefore, $c_{\omega}(F, E)=(F, E)$ and hence $X$ is a soft $\omega-T_{\frac{1}{2}}$-space.

Theorem 3.5. If the union of soft $\omega$-open sets is soft $\omega$-open set in a soft weak structure $\omega$ on a soft topological space, then every soft $\omega-T_{1}$-space is a soft $\omega-T_{\frac{1}{2}}$-space.

Proof. Suppose that $(X, \tau, E)$ is a soft $T_{1}$-space and the union of soft $\omega$-open sets is soft $\omega$-open set. It suffices to show that a set which is not soft $\omega$-closed also is not soft $g \omega$-closed set. Let $(F, E)$ is not soft $\omega$-closed. Let $x \in c_{\omega}(F, E)-(F, E)$. Then $(x, E) \tilde{\subset} c_{\omega}(F, E)-(F, E)$ and $(x, E)$ is a nonempty soft $\omega$-closed set in $X$ by Corollary 3.3. Hence, by Theorem 2.9, $(F, E)$ is not soft $g \omega$-closed.

The next example shows that the converse of the above theorem is not true in general.

Example 3.6. Let $X=\left\{h_{1}, h_{2}, h_{3}\right\}, E=\left\{e_{1}, e_{2}\right\}$ and $\tau=$ $\left\{\tilde{\emptyset}, \tilde{X},\left(F_{1}, E\right)\right.$, $\left.\left(F_{2}, E\right)\right\}$ where

$$
\begin{array}{llll}
F_{1}\left(e_{1}\right)= & \left\{h_{1}\right\}, & F_{1}\left(e_{2}\right)= & \left\{h_{1}\right\} ; \\
F_{2}\left(e_{1}\right)= & \left\{h_{1}, h_{2}\right\}, & F_{2}\left(e_{2}\right)= & X ;
\end{array}
$$

Let $\omega=\left\{\tilde{\emptyset},\left(F_{1}, E\right),\left(F_{2}, E\right)\right\}$ be an $\omega$ over $X$ with respect to $E$. Then $(X, \tau, E)$ is soft $\omega-T_{\frac{1}{2}}$-space but not soft $\omega-T_{1}$.

Theorem 3.7. Let $\omega$ be a soft weak structure on a soft topological space $(X, \tau, E)$. If $X$ is soft $\omega-T_{0}$, then for each $x, y \in X$ such that $x \neq y$, we have $c_{\omega}(x, E) \tilde{\neq} c_{\omega}(y, E)$.

Proof. Let $X$ be a soft $\omega-T_{0}$ and $x, y \in X$ such that $x \neq \tilde{y} y$. Then there exists soft $\omega$-open set $(F, E)$ such that $x \in(F, E)$ and $y \notin(F, E)$. Therefore $(F, E)^{\prime}$ is soft $\omega$-closed set such that $x \notin$ $(F, E)^{\prime}$ and $y \in(F, E)^{\prime}$. Since $c_{\omega}(y, E)$ is the intersection of all soft $\omega$-closed subsets that contain $y$, then $c_{\omega}(y, E) \tilde{\subset}(F, E)^{\prime}$ and hence $x \notin c_{\omega}(y, E)$. Thus $c_{\omega}(x, E) \tilde{\neq} c_{\omega}(y, E)$
Corollary 3.8. If $c_{\omega}(x, E)$ is a soft $\omega$-closed set for each $x \in$ $X$, and if for each distinct $x, y \in X, c_{\omega}(x, E) \tilde{\neq} c_{\omega}(y, E)$, then $X$ is soft $\omega-T_{0}$.

Proof. For each distinct $x, y \in X$, since $c_{\omega}(x, E) \tilde{\neq} c_{\omega}(y, E)$, there exists some $z \in X$ such that $z \in c_{\omega}(x, E)$ and $z \notin$ $c_{\omega}(y, E)$. If $x \in c_{\omega}(y, E)$, then $c_{\omega}(x, E) \tilde{\subset} c_{\omega}(y, E)$ which is a contradiction since $z \notin c_{\omega}(y, E)$. Thus $\left(c_{\omega}(y, E)\right)^{\prime}$ is soft $\omega$-open set such that $x \in\left(c_{\omega}(y, E)\right)^{\prime}$ and $y \notin\left(c_{\omega}(y, E)\right)^{\prime}$. Hence $X$ is soft $\omega-T_{0}$.

\section{Conflict of Interest}

No potential conflict of interest relevant to this article was reported.

\section{References}

[1] D. Molodtsov, "Soft set theory- first results," Computer and Mathematics with Applications, vol. 37, no. 4-5, pp. 19-31, 1999. https://doi.org/10.1016/S0898-1221(99) 00056-5

[2] P. K. Maji, R. Biwas and R. Roy, "Soft set theory," Computers \& Mathematics with Applications, vol. 45, no. 4-5, pp. 555-562, 2003. https://doi.org/10.1016/S08981221(03)00016-6

[3] D. Pie and D. Miao, "Form soft sets to information systems," in Proceedings of IEEE International Conference on Granular Computing, Beijing, China, 2005, pp. 617621. http://doi.org/10.1109/GRC.2005.1547365

[4] M. Shabir and M. Naz, "On soft topological spaces," Computers and Mathematics with Applications, vol. 61, no. 7, pp. 1786-1799, 2011. https://doi.org/10.1016/j.camwa. 2011.02 .006

[5] W. K. Min, "A note on soft topological spaces," Computers and Mathematics with Applications, vol. 62, no. 9, pp. 3524-3528, 2011. https://doi.org/10.1016/j.camwa.2011. 08.068

[6] I. Zorlutuna, M. Akdag, W. K. Min and S. Atmaca, "Remarks on soft topological spaces," Annals of Fuzzy Mathematics and Informatics, vol. 3, no. 2, pp. 171-185, 2012.

[7] B. P. Varol and H. Aygun, "On soft Hausdorff spaces," Annals of Fuzzy Mathematics and Informatics, vol. 5, no. 1, pp. 15-24, 2013. 
[8] N. Levine, "Generalized closed sets in topology," Rendiconti del Circolo Matematico di Palermo, vol. 19, no. 1, pp. 89-96, 1970.

[9] N. Cagman, S. Karatas and S. Enginogln, "Soft topology," Computers and Mathematics with Applications, vol. 62, no. 1, pp. 351-358, 2011. https://doi.org/10.1016/j.camwa. 2011.05 .016

[10] S. Roy and T. K. Samanta, "An introduction of a soft topological spaces," in Proceeding of UGC Sponsored National Seminar on Recent Trends in Fuzzy Set Theory, Rough Set Theory and Soft Set Theory, Howrah, India, 2011, pp. 9-12.

[11] A. Csaszar, "Generalized open sets," Acta Mathematica Hungarica, vol. 75, no. 1-2, pp. 65-87, 1997. http://doi. org/10.1023/A:1006582718102

[12] A. Csaszar, "Generalized topology, generalized continuity," Acta Mathematica Hungarica, vol. 96, no. 4, pp. 351-357, 2002. http://doi.org/10.1023/A:1019713018007

[13] A. Csaszar, "Generalized open sets in generalized topologies," Acta Mathematica Hungarica, vol. 106, no. 1-2, pp. 53-66, 2005. http://doi.org/10.1007/s10474-005-0005-5

[14] A. Csaszar, "Further remarks on the formula for $\gamma$-interior," Acta Mathematica Hungarica, vol. 113, no. 4, pp. 325332, 2006. http://doi.org/10.1007/s10474-006-0109-6

[15] A. Csaszar, "Remarks on quasi-topologies," Acta Mathematica Hungarica, vol. 119, no. 1-2, pp. 197-200, 2008. http://doi.org/10.1007/s10474-007-7023-4

[16] A. Csaszar, "Weak structure," Acta Mathematica Hungarica, vol. 131, no. 1-2, pp. 193-195, 2010. http://doi.org/10. 1007/s10474-010-0020-z

[17] A. Al-Omari and T. Noiri, "A unified theory of generalized closed sets in weak structure," Acta Mathematics Hungarica, vol. 135, no. 1-2, pp. 174-183, 2011. http://doi.org/10.1007/s10474-011-0169-0
[18] F. Feng, Y. B. Jun and X. Z. Zhao, "Soft semirings," Computer and Mathematics with Applications, vol. 56, no. 10, pp. 2621-2628, 2008. https://doi.org/10.1016/j.camwa. 2008.05 .011

[19] M. I. Ali, F. Feng, X. Liu, W. K. Min and M. Shabir, "On some new operations in soft set theory," Computer and Mathematics with Applications, vol. 57, no. 9, pp. 15471553, 2009. https://doi.org/10.1016/j.camwa.2008.11.009

[20] A. H. Zakari, A. Ghareeb and S. Omran, "On soft weak structure," Soft Computing, vol. 21, no. 10, pp. 2553-2559, 2017. https://doi.org/10.1007/s00500-016-2136-8

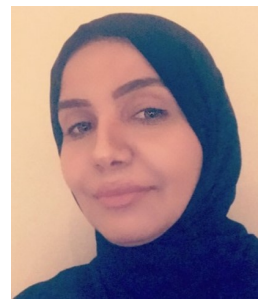

Hanan S. Al-Saadi is currently an associate Professor in Umm Al-Qura University. She received the Ph.D. degree in Pure Mathematics. Her primary research areas are Fuzzy topology and General topology.

Phone: +966-012-5472104

E-mail: hasa112@hotmail.com

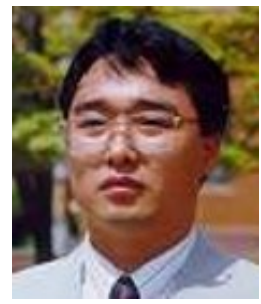

Won Keun Min is currently a professor in Kangwon National University. Chunchon, Korea. His main research interests include fuzzy topology and general topology.

Phone: +82-33-250-8419

Fax: +82-33-252-7298

E-mail:wkmin@cc.kangwon.ac.kr 